\section{VORWORTE}

04 Dr. med. Bernd Ramme, 1. Vorsitzender der DAA e.V.

05 Dr. med. univ. Olivia Krammer-Pojer,

1. Vorsitzende der OGKA

06 Dr. med. Andreas Wirz-Ridolfi, SACAM

08 Dr. med. Michael Weber und

Univ.-Prof. Prof. h.c. DI Dr. Dr. Gerhard Litscher,

Präsidenten der ISLAtranscontinental

\section{AUS DEN VERBÄNDEN}

41 DAA e.V.: Aktuelles und Termine

48 SACAM: Aktuelles und Termine

49 OGKA: Aktuelles und Termine

53 ISLA: Aktuelles und Termine

\section{ZAA RUBRIKEN}

09 Neues, Wissenswertes, Kurioses

47 DAA Kalender

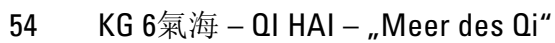

\title{
AUS DER FORSCHUNG
}

TCM - FREQUENZEN

Manfred Reininger

20 Neues aus der Mitte - „Musikalisches“ Vorgehen in Bezug zur "Mitte“

News from the middle - musical proceeding relating to the middle

\section{AUS DER PRAXIS}

\section{TCM}

Olivia Krammer-Pojer

10 Schwangerschafts-Guide der chinesischen Medizin oder "Die Erziehung des Fetus"

Pregnancy-guide of chinese medicine - or "fetal education"

TCM - AKUSTIK

Johannes Hickelsberger

16 Die 3 Säulen der Tinnitus-Therapie

Three pillars of tinnitus therapy

TCM - FREQUENZEN

Anton Keppel

Die Informationen der Autoren zu Diagnostik und Therapie sind teilweise Neuland und decken sich nicht immer mit den Kursinhalten der DAA. Sie sind als Anregung gedacht und geeignet, eigene Überprüfungen zu fördern und einen offenen Dialog zu führen. Ihre Ideen, Ergebnisse oder Meinungen, falls sie für alle Mitglieder interessant sein könnten, veröffentlichen wir gerne unter der neuen Rubrik „Nachlese“ im jeweiligen Folgeheft.

\section{TITELTHEMA}

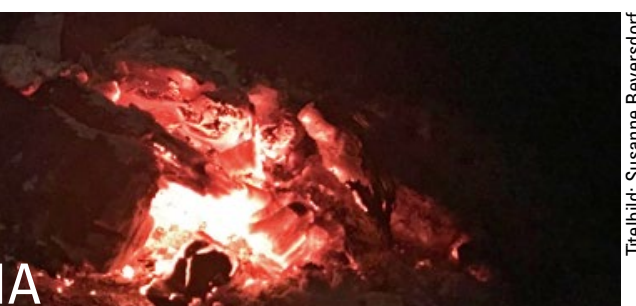

Silent inflammation / Seite 28

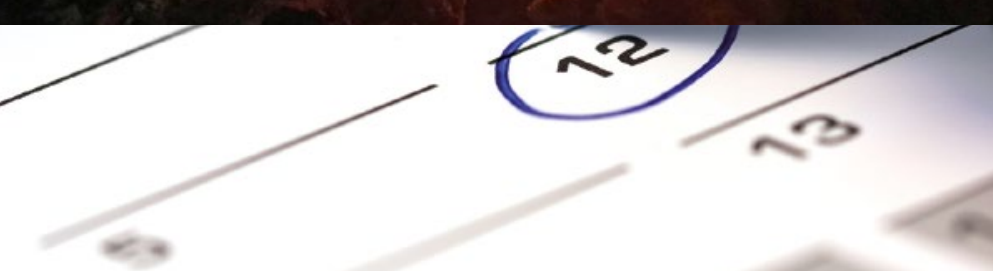

TERMINE | ab Seite 41

\section{SCHLUSSPUNKT}

KG 6氣海 - OIHAI"MEER DES Q1" $\mid$ ab Seite 54 